\title{
« RÉGLONS-LUI SON CAS ». PSYCHANALYSE, RÉCITS CLINIQUES, ENJEUX
}

Thamy Ayouch

EDK, Groupe EDP Sciences | « Psychologie Clinique »

2017/2 n 44 | pages 99 à 114

ISSN $1145-1882$

ISBN 9782759822089

Article disponible en ligne à l'adresse :

https://www.cairn.info/revue-psychologie-clinique-2017-2-page-99.htm

\section{Pour citer cet article :}

Thamy Ayouch, « «Réglons-lui son cas ». Psychanalyse, récits cliniques, enjeux », Psychologie Clinique 2017/2 ( $\left.\mathrm{n}^{\circ} 44\right)$, p. 99-114.

DOI 10.1051/psyc/20174499

Distribution électronique Cairn.info pour EDK, Groupe EDP Sciences.

(C) EDK, Groupe EDP Sciences. Tous droits réservés pour tous pays.

La reproduction ou représentation de cet article, notamment par photocopie, n'est autorisée que dans les limites des conditions générales d'utilisation du site ou, le cas échéant, des conditions générales de la licence souscrite par votre établissement. Toute autre reproduction ou représentation, en tout ou partie, sous quelque forme et de quelque manière que ce soit, est interdite sauf accord préalable et écrit de l'éditeur, en dehors des cas prévus par la législation en vigueur en France. Il est précisé que son stockage dans une base de données est également interdit. 


\section{We Écrire le cas. Du récit à la critique}

Thamy Ayouch ${ }^{[1]}$

\section{Résumé}

À partir d'une inversion de la question du récit de cas, cherchant à voir ce que la présentation de cas peut révéler d'une certaine psychanalyse plutôt que ce que celle-ci peut dire du cas clinique, l'auteur se propose d'aborder certaines pratiques de l'écriture clinique, pour réfléchir à la spécificité de l'écriture de cas en psychanalyse. Est ainsi mise en exergue la violence commune exercée contre la singularité clinique, consistant à l'inscrire dans une théorisation qui la précède et dont elle n'est qu'une confirmation, au mieux un « exemple éclairant ». Apparaissent alors les questions de l'occultation théorique et (contre)transférentielle de la clinique, de la valeur d'usage du cas clinique dans la théorisation, de l'excès d'imaginaire filtrant dans certaines interprétations de cas et de la manière dont y joue ou non l'analyse du transfert. Soulevant les question de l'exemplarité du cas, sa présentation comme paradigme d'une structure nosographique sous-jacente, sa visée "pédagogique ", son inclusion dans une psychopathologie, l'auteur interroge la manière dont opère la " vérité historique » du cas et le statut de la narrativité dans l'écriture de cas.

Mots clés

Écriture de cas ; critique ; usage du cas ; vérité historique ; violence clinique et thorium. Summary

\section{Writing Cases. From Narrativeness to Critique}

Reversing the usual case narrative perspective, and asking what some case studies may reveal about a certain psychoanalysis, rather than what psychoanalysis may say about cases studies, the author dwells on some aspects of clinical writing in order to analyse the specificity of case writing in psychoanalysis. He sets forth the common violence against clinical singularity aired by some case studies which conceive the case only as a confirmation, and at best a "shining example ", of a previous theory. He asks the questions of how theory and (counter)transference may obscure clinical situations, of the use value of clinical cases in theory, and of the excessive Imaginary that is conjured up in some case interpretations that neglect to analyse their (counter)transference position. He raises the questions of the exemplarity of cases, their use as paradigms of nosographic structures, their " pedagogic » aim, and their inclusion in a psychopathology. He finally questions the way " historical truth » may operate in a case, and how it may be dissociated from narrativeness when it comes to case writing.

Key words

Case use ; case writing; clinical and theoretic violence ; critique ; historical truth.

[1] Professeur, Université Paris Diderot, CRPMS, Psychanalyste Paris, thamy.ayouch@gmail.com 
oit la proposition d'une clinicographie, ainsi définie par Guénaël Visentini : “ champ de réflexivité sur les pratiques d'écriture clinique, à partir de leur ancrage socio-historique (groupes d'appartenance des scripteurs, état des controverses, normes et préjugés de la discipline, enjeux politiques) et de leurs contraintes "spécifiques" (épreuves cliniques d'un réel posant question) " ${ }^{[2]}$.

Je me propose, dans cet article, de situer une série de problèmes liés à la pratique d'écriture des cas, à travers quelques exemples d'écrits contemporains. C'est donc en partant d'une inversion de la question habituelle que j'ouvrirai la réflexion : il s'agira, plutôt que de chercher à saisir ce que la psychanalyse peut dire du cas clinique, de voir ce que la présentation de cas peut révéler de la psychanalyse, du moins d'une certaine psychanalyse.

\section{Wes emplois du cas: le lapin dans le chapeau}

Un article au titre éloquent, "La relation homosexuelle, une révélation divine dans une vie de chien", affirme, sans préambule ni ambages, sa thèse en guise de titre de première partie : "Toutes les femmes homosexuelles racontent la même chose : avec leur mère, ça n'a jamais passé” (Bourdelon, 2003, p. 95). Avant que de présenter la situation clinique examinée, l'interprétation généralisée est assénée en ces termes : là où le lien homosexuel primaire et secondaire à la mère a été défectueux, la rencontre amoureuse homosexuelle, à l'âge adulte - remplaçant ce lien ou poussant à l'addiction - se pérennise et ne passe plus, dans une relation qui " ne trouvera une nouvelle homéostasie narcissique qu'à se perversifier » (idem).

De qui s'agit-il? De Marine, quarante ans, née d'un accouchement catastrophique suivi d'une dépression post-partum, présentant, par « défaut de la fonction contenante maternelle » (ibid., p. 97), des comportements boulimiques. Fraichement séparée de son mari, elle ne lui était liée, écrit-on, que par un faux-self hyperadapté. De son avortement ne la sauve qu'un mariage, et pour fuir cette union, écrit-on, elle s'accroche à son fils décrit en ces termes : “ l'enfant choyé par sa mère devient un "pédé" (il est castré), une larve comme le mari. L'enfant, objet phallique, complète le narcissisme maternel » (ibid., pp. 97-98).

La relation homosexuelle, au “ potentiel psychotisant » (ibid., p. 105), correspond à une « néo-réalité, $[\ldots]$ ghettoïsation, [...] néo-identité sexuelle confortée par la spécularité apportée par la compagne, [qui] répondent alors à une crise existentielle majeure » (ibid., p. 111). L'avidité orale que recèle le lien primaire carencé avec la mère a raison des rapports passionnels de Marine ${ }^{[3]}$, condamnés à l'échec, car « la fonction paternelle n'ayant pu être introjectée, l'Edipe n'ayant pas majoritairement

[2] Voir son introduction au numéro.

[3] « Grâce à l'objet externe homosexuel, l'objet interne est réanimé (il était auparavant contre-investi et/ou désinvesti). Pourtant la destructivité de l'avidité orale, réclamant un investissement massif, va à l'encontre du besoin de petites doses. La liaison amoureuse passionnée porte alors en elle sa fin nécessaire » (Bourdelon, 2003, p. 100). 
structuré la psyché, il faut toujours une "porte de sortie" à l'investissement massif enfermant et fusionnel » (ibid., p. 102).

Pourquoi l'opium fait-il dormir? Parce qu'il possède une vertu dormitive ${ }^{[4]}$. Pourquoi cette patiente souffre-t-elle? Parce qu'elle est homosexuelle, id est, présente un Edipe non résolu, un déni de la différence des sexes et donc de la différentiation des individus, de la distinction entre objet interne et objet externe, et une conséquente régression à un monde ante-œdipien qui porte en son sein la destructivité. Le choix d'objet homosexuel est cause et raison de tous ses troubles : il rend manifeste sa faille narcissique. La vérité du cas tient dans sa réduction à cette clé de voûte explicative, unification bien tempérée de sa multiplicité, venant entériner l'orthodoxie théorique habituelle où l'homosexualité est choix du même, inaccession à la triangulation, à l'CEdipe et à la différence.

Dans un autre article affichant une perspicace interrogation pour titre - "La relation homosexuelle est-elle une relation incestueuse?" (Gérard, 2003) - la visée est d'emblée claire : il s'agit d'établir une psychologie du développement généralisable, détaillant les aléas de l'évolution depuis l'homosexualité primaire, supposés déterminer le choix d'objet homosexuel adulte. En guise d'illustration, deux patient/es (homosexuelle féminine, homosexuel masculin, pourquoi chercher donc davantage ?) sont évoqués, Cécile et Pierre, tous deux invertis pour avoir échoué à s'extraire d'une homosexualité primaire. La faute en revient, sans nulle surprise, à la mère, responsable d'une séduction pré-œedipienne et incestueuse et de la fixation infantile qui en est résultée.

Cécile manifeste, soutient-on, une conflictualité avec l'objet primaire : elle recourt, dans une recherche antidépressive, à des unions avec des femmes qui la soumettent à leur emprise sado-masochiste (ibid., pp.114-115). La relation qu'elle établit et répète avec ses partenaires est incestueuse car duelle, non triangularisée ${ }^{[5]}$. Il en est de même pour Pierre, chez qui la personnalité dépressive de sa mère, à un moment de la première enfance et de la détresse infantile, a provoqué un vide de représentation (ibid., p. 127). Pour les deux, la "mère morte " est donc la cause de l'homosexualité : "Je pense que l'aspect abandonnique, "mère morte", serait central dans l'organisation d'une telle pathologie narcissique, laquelle n'aurait rien de spécifique au garçon ou à la fille. C'est la nature particulière de la relation à l'objet qui serait à l'origine d'une fixation à l'homosexualité ; celle-ci serait à entendre comme prise dans un processus, comme un arrêt sur un parcours » ${ }^{[6]}$.

[4] « Domandatur causam et rationem quare/Opium facit dormire [...] Quia est in eo/Virtus dormitiva/Cujus est natura/Sensus assoupire » (Molière, Le malade imaginaire, Acte III, scène 3).

[5] « Le couple narcissique mère-fille, comme isolé, reste hors d'atteinte de la fonction paternelle, favorisant ainsi la fixation infantile. Celle-ci ne pouvant que limiter le développement du psychisme, prédispose aux angoisses narcissiques et au sentiment d'insécurité. C'est ainsi que, pour la patiente, s'est développé le désir inconscient d'être et de rester en relation avec des femmes omnipotentes, ce qu'elle réalisera dans sa vie adulte par son homosexualité » (Gérard, 2003, p. 123).

[6] Gérard, 2003, p. 127. 
Outre cette préoccupante pathologisation de l'homosexualité, c'est ici la visée d'étiologie généralisée au mépris de la singularité de ces situations, qui semble bien contestable.

Ces deux exemples de récits de cas montrent la violence ordinaire exercée contre la singularité clinique, consistant à l'inscrire dans une théorisation évolutionniste qui la précède et dont elle n'est qu'une confirmation, au mieux un exemple éclairant. Apparaissent deux questions, celle de l'occultation théorique et contre-transférentielle de la clinique, mais aussi celle de la valeur d'usage du cas clinique dans la théorisation. La question de l'homosexualité, ramenée implicitement à une normation du sexuel désigne, à travers un verre grossissant, la maltraitance exercée à l'endroit de la clinique par:

- l'oubli de la singularité du sujet, en faveur d'une théorie généralisée et un désir d'universaliser le cas, devenu catégorie ;

- le généreux partage, par l'analyste, sinon avec ses analysant/es, du moins avec ses lecteurs/trices, de ses fantasmatisations peu analysés au sujet des père, mère, homme, femme et autres catégories de ses théories sexuelles infantiles. Si ces fantasmatisations peuvent s'avérer centrales lorsqu'elles sont analysées dans leur charge imaginaire contre-transférentielle, elles n'ont pour vocation ni d'être dressées en théories, ni d'être assénées aux analysant/es.

Plus qu'une enluminure de la théorisation, le motif de l'homosexualité du cas clinique peut aussi devenir véritable iconographie. La "Psychogénèse d'un cas d'homosexualité féminine" de Freud a fait l'objet de diverses analyses ${ }^{[7]}$ : elle apparaît, chez bien des auteur/es, comme cas parangon de l'homosexualité du moins féminine, sinon universelle, soulevant ainsi la question de savoir si un cas peut-être paradigmatique de quoi que ce soit. L'appropriation de cas canoniques fait ainsi apparaître plus clairement la question de savoir quelle nouveauté la clinique et ses réinterprétations convoquent.

Pour un auteur, c'est l'ironie de la “ jeune homosexuelle » qui prend valeur, généralisée, à la fois de condition étiologique et de conséquence de l'homosexualité (Barazer, 1999). Venant, dans la formule “ Ah, mais c'est très intéressant ! », servir la résistance de la patiente et signifier à Freud qu'elle n’adhère pas à ses constructions - ce qui somme toute reste légitime pour tout/e patient/e face à une interprétation invasive de l'analyste - l'ironie est conçue comme impossible dépassement d'une désillusion portant sur la crédibilité de la parole paternelle (ibid., p. 105). Elle présente ce paradoxe de comporter un désaveu de l'énoncé sans énoncé de désaveu (ibid., p. 107).

Après avoir rappelé les considérations de Freud à l'endroit de la cure commandée - en omettant toutefois de souligner que, pour Freud, celle-ci n'est en aucun cas malade - l'auteur poursuit :

[7] Diverses analyses mettent en exergue sa sexuation du côté masculin (Melman, 2002), son désistement comme dette ou culpabilité (Pickman, 2010), ou encore comme posture vengeresse (Pommier, 2002). 
"Puis il [Freud] ajoute que, chez un pervers, c'est la cure elle-même qui peut se trouver investie d'une dimension de défi : le pervers y participerait d'autant plus consciencieusement et obéirait d'autant plus docilement à la règle fondamentale qu'il démontrerait par là même que le processus ne pourrait aucunement avoir prise sur lui, entamer son « moi ». C'est ce "plan secret » qui peut amener ces patients à la psychanalyse sous couvert d'une demande de normalisation de leur conduite sexuelle [...]. C'est l'expérience analytique tout entière qui se trouve ainsi détournée à l'usage de preuve, à l'appui du désaveu de la castration. Nous retrouvons donc là, à nouveau, cet acquiescement qui réaliserait un désaveu et que Freud associe à un fonctionnement pervers, voire même à une sorte de manipulation délibérée „ ${ }^{[8]}$.

Étrange approximation réalisée ici entre l'homosexualité de la jeune patiente, son ironie et la perversion, là où, dans la description freudienne en question ${ }^{[9]}$, il n'est fait nulle mention de perversion ou fonctionnement pervers, d'échec de processus de prise sur soi, d'entame du moi, de demande, par la patiente, de normalisation de sa conduite sexuelle, de désaveu de la castration ou de manipulation délibérée. Nombre d'éléments de la lecture de cet auteur, absents du texte freudien, semblent correspondre à une projection après-coup de catégories ultérieures, développées à partir de l'étude freudienne du modèle circonscrit du fétichisme ou de la généralisation lacanienne et post-lacanienne de ce modèle comme clé psychique de la perversion.

L'ironie, forme de langage et d'humour, s'avère donc perverse ${ }^{[10]}$. Le défi lancé au dogmatisme, la dérision devant l'ontologie et la sédition de l'ironie sont alors à

[8] Barazer, 1999, p. 108.

[9] "La jeune fille n'était pas une malade - elle ne souffrait pas pour des raisons internes, elle ne se plaignait pas de son état - et la tâche commandée ne consistait pas à résoudre un conflit névrotique, mais à faire passer l'une des variantes de l'organisation sexuelle génitale dans l'autre de ces variantes. Cette opération, la suppression de l'inversion génitale ou homosexualité, ne s'est jamais présentée, d'après mon expérience, comme chose facile. Bien plutôt, j'ai trouvé qu'elle ne réussit que dans des circonstances particulièrement favorables et que même alors le succès consiste essentiellement en ce qu'on a pu, pour la personne confinée dans l'homosexualité, dégager la voie jusqu'alors barrée menant à l'autre sexe, donc rétablir pour cette personne la fonction bisexuelle complète. Libre à elle alors de laisser désaffectée l'autre voie bannie par la société, comme cela s'est produit effectivement dans quelques cas individuels. II faut se dire que la sexualité normale elle aussi repose sur une restriction du choix d'objet, et qu'en général transformer un homosexuel pleinement développé en un hétérosexuel est une entreprise qui n'a guère plus de chances d'aboutir que l'opération inverse, sinon que, pour de bonnes raisons pratiques, cette dernière n'est jamais tentée. [...] En général, l'homosexuel se montre incapable d'abandonner son objet de plaisir [...]. Si jamais il se soumet à un traitement, c'est qu'il y a été poussé par des motifs principalement extérieurs, à savoir les inconvénients et les dangers sociaux de son choix d'objet, et que ces composantes de la pulsion d'autoconservation s'avèrent trop faibles dans le combat contre les tendances sexuelles. Et bientôt, on peut découvrir son plan secret, qui était d'utiliser l'échec éclatant de cette tentative afin de se tranquilliser l'esprit : il aurait fait tout ce qui était possible contre ses mœurs particulières, et pourrait donc s'y abandonner avec bonne conscience. Là où la tentative de guérison a été motivée par le souci de ménager des parents et un entourage affectionnés, le cas est un peu différent. Alors on est en présence de tendances réellement libidinales qui peuvent développer des énergies contraires au choix d'objet homosexuel, mais dont la force est rarement suffisante. [...] Pour toutes ces raisons j'évitai absolument de laisser espérer aux parents l'accomplissement de leur désir » (Freud, 1920/1973, pp. 249-250).

[10] "Dans notre expérience des cures où cette dimension de l'ironie comme symptôme nous est apparue de façon particulièrement insistante et problématique, il nous a semblé pouvoir chaque fois retrouver dans l'histoire de ces patients quelque chose de l'ordre, effectivement, d'une désillusion cautionnée par la réalité événementielle, à un moment clef de leur histoire. [...] La défiance est "agie", mise en acte par le contexte énonciatif, tandis que, simultanément, la confiance est "dite" par la 
corriger : l'analyste vise à les redresser en rappelant au sérieux de la réalité et à son univocité, et à bannir l'humour, la double-entente, ou même la confusion irréductible du langage, sommé de devenir transparent. Et au fondement de la perversion du langage propre à l'ironie, se tient, assurément, la perversion de l'homosexualité.

\section{We Questions et problématisations}

Ces expositions de cas d'homosexualité, qu'il s'agisse de situations cliniques rencontrées par ces analystes ou de cas classiques revisités, font surgir un ensemble de questions.

1. Pourquoi donc ces récits de cas dérangent-ils? L'excès d'imaginaire filtrant dans ces interprétations doublées d'une pathologisation irréductible de l'homosexualité semble faire de l'analyste un/e montreur/se d'ours. La procédure du traitement interprétatif du cas consiste ici à ramener la complexité psychique à la simplicité d'un trait (l'homosexualité) ou d'un mécanisme (le désaveu) pour rendre compte unitairement d'une expérience et d'une structure. L'interprétation vaut comme « injure diagnostique " ${ }^{[1]}$, sentence sans appel. Privant les principaux/ales concerné/es de toute capacité symbolique, en exigeant presque une confession de leur ignorance, elle prétend extraire une vérité de leurs désirs et de leurs corps ramenée à des étiologies généralisables.

2. Une deuxième interrogation consiste à demander si un cas peut servir de paradigme d'une structure psychopathologique sous-jacente. À travers l'étude d'un cas unique sont souvent visées des étiologies susceptibles d'être généralisées pour établir une psychopathologie des structures. Ce traitement de l'observation clinique demeure voisin de l'utilisation du cas en psychiatrie: il aboutit à l'érection d'un modèle typologique. Il semble alors légitime de demander si l'étude de cas en psychanalyse peut avoir pour objectif une psychopathologie, et comment elle demeure alors psychanalytique. Que conserve d'analytique ici une posture pédagogique, démonstrative, objectivante, procédant d'un exercice psychiatrique?

3. Se pose également la question de l'exposition du cas. Quel rapport peut-on, lecteurs/lectrices, entretenir avec la vie d'un/e analysant/e, appréhendé/e, de surcroît hors du transfert propre à une cure? Nous pénétrons l'intimité d'une relation dans laquelle, malgré que l'analyste en ait, l'analysant/e ne nous a pas convié/es. Comment ne pas friser ici le voyeurisme? Comment accepter que, d'une expérience à deux, on rende compte, étonnamment, par un écrit singulier? Que serait le cas écrit par

reprise en écho de la parole "paternelle". Ces deux positions ne se conflictualisent jamais. [...] Cette coexistence de deux niveaux, l'un véhiculant le désaveu, l'autre la reconnaissance, mais sans que cette reconnaissance ne soit " modalisée ", constamment à l'œuvre dans les stratégies discursives de l'ironiste, s'apparente, selon nous, à cette déchirure du moi caractéristique de la perversion, dont Freud fait état à propos du fétichisme, bien plus que d'autres formes de non-créance répondant, par exemple, à l'isolation obsessionnelle. L'ironie serait une perversion du rapport à la parole » (Barazer, 1999, pp. 117-118).

[11] Ayouch, 2015. 
l'analysant/e ? Si le récit de cas est à intégrer dans une analyse du transfert, le désir de l'analyste y occupe le premier plan. Mais c'est une réalité que bien des analystes oublient lorsqu'ils font du transfert une répétition ne concernant que l'analysant/e. 4. Cette interrogation sur les conséquences éthiques du récit de cas se prolonge eu égard à la double règle fondamentale de l'analyse. En effet, le principe de l'association libre semble mis à mal dans le geste totalisateur, synthétique, du cas unifié. La règle d'abstention est ici également atteinte : l'analyste intervient activement hors de la cure et de la réalité psychique de l'analysant/e, en publiant le cas. En outre, l'interrogation éthique mobilise ici une réflexion sur le contrat analytique. Par-delà le cadre et les règles techniques à tenir, l'éthique de l'analyse reste tributaire de ce qui est défini par un contrat passé entre analyste et analysant/e, décidant de la fréquence des séances, de la visibilité de l'analyste, du cadre, du prix, des modalités concernant les séances ratées, etc. Le contrat institue tout autant l'analysant/e et l'analyste, il vient régler les droits de l'analyste, mais aussi ses obligations et leurs limites. Nulle interprétation de l'analyste ne saurait porter sur un contrat sur lequel il/elle ne se soit au préalable mis/e d'accord avec l'analysant/e. Les lecteurs/trices ou auditeurs/ trices du cas ne font pas partie de ce contrat : la confiance, nécessaire à son établissement, suppose que l'analysant/e soit assuré/e qu'il n’y aura pas de tiers réel dans la "situation analytique ". Sauf à en décider d'avance, ce qui ne manquerait pas d'installer un type particulier de transfert, mobilisant alors une analyse particulière. 5. Pour approfondir la réflexion sur les conséquences éthiques mais aussi épistémologiques du récit de cas, je mettrai ici en exergue, par une analyse pragmatique, le changement de places qu'opère l'écriture du cas. Dans le dispositif analytique, le/a locuteur/trice est essentiellement l'analysant/e. L'analyste occupe la place d'interloctueur/trice, et d'allocutaire. Si l'analysant/e est le/a délocuté/e, le/a destinataire reste l'autre, les autre, et le grand Autre. Dans le récit de cas, le/a locuteur/trice est l'analyste; l'analysant/e, ni interlocuteur/trice ni allocutaire, n'est plus que délocuté/e. Les allocutaires et destinataires semblent se confondre ici, qu'il s'agisse de superviseur/se, de psychanalystes confrères et consœurs, du public lors d'un exposé, de lecteurs/trices ou d'étudiant/es dans un contexte académique. Il semble alors pertinent de se demander ce qui se passe lorsque l'analysant/e prend connaissance d'un récit de son cas, en devient le/a destinataire sans en être l'allocutaire. La question porte autant sur le secret et l'obligation de réserve de l'analyste, que sur les réactions affectives susceptibles d'être provoquées chez l'analysant/e qui se reconnaît dans le récit de cas. Outre le sentiment d'inquiétante étrangeté de ne pas retrouver ce que le transfert lui a fait vivre de son côté, c'est probablement à une dépossession qu'il/elle peut avoir affaire, une confiscation de son histoire par un/e autre, sujet supposé savoir, manifestant une autorité en la matière - et que la publication confirme dans cette autorité. L’invariance de l'écrit, son immutabilité - contre la perlaboration, l'évolution pendant et après l'analyse, ou tout simplement l'oubli peut fixer ici une identité qui devient alors rigide et définitive. Se pose en outre la 
question de savoir quel effet sur le transfert la publication d'un cas peut avoir indépendamment de sa prise de connaissance par l'analysant/e - lorsque la cure est poursuivie après cette publication. Plus encore, même lorsqu'il ne s'agit pas d'elle/de lui, pour un/e analysant/e, lire le compte rendu d'une autre analyse que la sienne faite par son analyste peut produire une véritable inflation fantasmatique. La/le voici devenu/e voyeur/se d'une relation que son analyste a entretenue avec un/e autre, développant le fantasme d'une autre connaissance de l'analyste, et, de fait, confronté/e aux réactions et affects de l'analyste.

6. Cette question de publication, ou plus précisément de publicisation soulève d'autres interrogations. "Ce qui se dénomme cas - écrit Fédida, dans quelque contexte que l'on se situe - doit recevoir statut de présentation publique. Sinon, ce n'est pas un cas " (Fédida, 1995, p. 258). C'est dire ici que c'est son adresse et ses destinataires qui font le cas, et non la singularité ou l'originalité de l'histoire rapportée par ce cas. Si le compte rendu d'un cas vaut souvent dans la formation de l'analyste, le cas est-il à publiciser davantage que dans l'école d'analyse ? Si le public, analytique dans le cadre de la présentation de cas, est supposé rester restreint, force est d'admettre que les supports de publication (revues, livres, manuels) connaissent une diffusion bien plus étendue, et l'analysant/e reste toujours susceptible de s'y rencontrer. Une solution alternative à la présentation de cas de sa propre clinique est de reprendre ceux d'autres analystes. Historiquement, les cas célèbres de la psychanalyse - les Cinq psychanalyses de Freud, les cas Aimée de Lacan, Dick de Mélanie Klein, ou Piggle de Winnicott, pour ne citer que ceux-là - constituent autant de balises pour une communauté analytique. Ils paraissent davantage susceptibles d'être exposés, peut-être parce que l'analysant/e y est à ce point stylisé/e qu'il/elle semble ne plus exister en réalité, et se rapprocher d'une abstraction. Si la lecture de ces cas peut contribuer à une transmission intergénérationnelle de la psychanalyse, il demeure pertinent de se demander à quoi cela tient, quelle valeur ils recèlent actuellement, quels restes de savoir ils contiennent et comment on y a accès, hors du transfert.

7. À radicaliser ces questions, l'on pourrait demander si la psychanalyse est véritablement susceptible de produire des cas. Plus précisément, que permet ici le récit, qu'est-ce qu'il écarte, lorsque le cas a été déformé pour préserver l'intime de la cure? Ne pêche-t-il alors pas par incomplétude? Peut-on restaurer quelque chose du transfert et de la spécificité du déroulement d'une cure par la linéarité d'un récit? Si l'on renonce à l'idée que le cas restaure la vérité de l'analysant/e, capture-t-il du moins la vérité de la situation analytique? Que penser, en outre, d'histoires de cas exemplaires, articulant, le long de la règle classique des trois unités du théâtre (action, vraisemblance et bienséance), une présentation des personnages, une intrigue et un dénouement? Quelle romanticisation de la cure mettent-elles en place? Plus encore, pourquoi rendre compte d'une analyse, devant qui, à quelle fin et de quelles façons? 8. Se repose alors la question des destinataires du cas : s'agit-il de psychanalystes au sein d'une société ou école d'analyse, d'un public large et indifférencié de 
lecteurs/lectrices, ou d'un ensemble d'étudiant/es de psychologie à former? Dans ce dernier contexte académique, le récit de cas ne manque pas d'interroger le statut de la psychanalyse à l'université. Que faisons-nous, universitaires praticiens de la psychanalyse, lorsque nous enseignons de la psychopathologie, et dans quelle mesure utilisons-nous nos cas? Apparaît ici la question de la visée pédagogique des cas : servent-ils à enseigner, illustrer, incarner, ou démontrer quoi que ce soit? Je tenterai d'aborder maintenant quelques-unes des difficultés ici soulevées.

\section{WE L'éthique, par-delà le juridique}

Pour répondre à certaines de ces interrogations éthiques, Dominique Thouvenin propose de considérer le cas non point comme présentation d'éléments biographiques identifiables, mais comme support d'une information de type scientifique (Thouvenin, 1999, p. 13). L'intérêt du cas ne tient alors pas, selon l'auteure, à la singularité des traits qu'il met en avant, mais à l'apparition de processus psychiques permettant “ la différentiation en paranoïa, hystérie, névrose obsessionnelle...» (Thouvenin, 1999, p. 13). De prime abord, cette présentation du cas comme illustration d'une structure soulève à nouveau la question de sa visée (serait-il une « vignette clinique »?), et davantage encore à l'ère d'une psychanalyse non psychopathologique. Cette anonymisation du cas, souligne l'auteure, va par-delà la singularité de tel/le ou tel/le analysant/e : " le cas est une observation qui valide ou invalide une théorie, ce qui suppose un cadre théorique préexistant construit dans lequel il va s'insérer ", et “ l'analyste n'est plus dans la relation de droit privé dans laquelle s'inscrit son rapport avec l'analysé, mais est confronté aux règles du débat scientifique et au caractère public de ce dernier " ${ }^{[2]}$. Et Dominique Thouvenin de conclure : “De ce point de vue, un parallèle intéressant peut être établi entre l'utilisation des matériaux des traitements psychothérapeutiques sous forme de publication dans les revues psychanalytiques et les commentaires dont les arrêts sont l'objet dans les revues juridiques » ${ }^{[13]}$.

Cette précédence de la théorie, la visée, somme toute nosographique, du cas, et son inscription dans une scientificité juridique ne sont pas sans poser des problèmes. Est ici supposée une casuistique psychanalytique, mise en perspective de plusieurs cas permettant l'établissement d'une jurisprudence clinique, d'une classification et d'une direction de la cure. En outre, cette juridification du médical, où la nosographie est comparée à une casuistique juridique, doublée d'une médicalisation du juridique, où la casuistique juridique se verrait prêter, à terme, un effet thérapeutique, n'est pas sans évoquer le pouvoir de normalisation propre à l'expertise médicale défini par Foucault (1999).

[12] Thouvenin, 1999, p. 14.

[13] Thouvenin, 1999, p. 14 
Si le cas est à l'abri de toute attaque juridique (et cette immunité juridique, du reste, ne garantit en rien ici une réflexion et un questionnement éthique), c'est pour les qualités d'abstraction qu'il présente : comme dans une décision en justice, ce n'est pas, selon l'auteure, l'individu singulier qui est en cause dans le cas en psychanalyse, mais sa qualité clinique ou juridique (Thouvenin, 1999, p. 19). Il semble judicieux de se demander si, la psychanalyse étant une clinique de l'hypersingularité, il est possible de dissocier ici un individu de sa qualité clinique. L'auteure reconnait toutefois une légère distinction entre les cadres analytique et juridique : " le cas est produit à partir d'un analysant selon la grille interprétative établie par l'analyste, tandis que le commentaire porte sur une décision dont les conditions de production sont totalement extérieures à celui qui la commente »(Thouvenin, 1999, p. 24). Différence de taille, puisqu'il ne s'agit de rien moins que de l'implication contre-transférentielle de l'analyste qui, comme souligné auparavant, s'avère ici centrale dans la production du cas.

Si la juridification des récits de cas règle donc bien peu les questions d'éthique, elle n'en laisse pas moins irrésolue la scientificité de l'exercice, dont l'abstraction sert, selon Dominique Thouvenin, à prémunir contre toute attaque juridique. Reste en effet posée la question de savoir comment l'on passe d'un cas singulier à une généralité, et quelle valeur a la nosographie, ou la psychopathologie, dans un récit d'analyse. Si la scientificité des sciences de la nature (ou sciences exactes) est fondée sur la vérifiabilité et la reproductibilité, le compte rendu d'analyse n'a alors rien de scientifique. Dans cette scientificité, le langage est un médium transparent, là où dans le compte rendu d'analyse, un autre espace discursif, à la fois narratif, historique, littéraire et herméneutique, semble s'ouvrir. Cela à la condition de définir ce compte rendu essentiellement comme institution d'une nouveauté et non point illustration d'un développement théorique pré-établi.

Dans la lecture des Cinq psychanalyses effectuée par Lacan, le rapport entre singularité et généralité est probablement défini à rebours de l'abstraction scientifique ici pointée par Dominique Thouvenin :

“Je crois vous voir démontré que Freud en est parti, que chaque fois il s'agit pour lui de l'appréhension d'un cas singulier, et c'est cela qui a fait le prix de l'analyse, de chacune de ces cinq grandes psychanalyses; les trois que nous avons déjà vues, élaborées, travaillées ensemble, vous le démontrent, c'est que c'est là qu'est vraiment l'essentiel, son progrès, sa découverte, dans la façon de prendre un cas dans sa singularité. Eh bien, le prendre dans sa singularité, qu'est-ce que ça veut dire? Cela veut essentiellement dire que pour lui l'intérêt, l'essence, le fondement, la dimension propre de l'analyse, c'est la réintégration par le sujet de son histoire jusqu'à ses dernières limites sensibles, c'est-à-dire jusqu'à quelque chose qui dépasse de beaucoup les limites individuelles " ${ }^{[14]}$.

[14] Lacan, 1975, p. 20. 
Le cas semble donc ouvrir vers autre chose qui y insiste. Je choisis de lire cet au-delà de l'individualité du cas comme stratification historique des temps de l'analysant/e, de l'analyste, et de l'institution analytique (historicité des modèles, des théorisations et des formations). La singularité du cas est ce qui contient une multiplicité d'histoires unifiées en apparence. Reste toutefois posée ici la question de savoir ce qu'apporterait la publication d'un cas à cette réintégration par le sujet de son histoire. Force est d'admettre que ce n'est pas tant l'histoire de ce sujet qui est ici au centre, que celle de la psychanalyse, de sa recherche et de sa transmission. Et celle de l'analyste.

Une première objection éthique peut toutefois être ici écartée : celle d'une instrumentalisation des analysant/es pour la recherche en psychanalyse et sa transmission. À cela, il conviendrait de répondre qu'assurer le contexte clinique de la cure n'est pas seulement une visée éthique en sus de la recherche : elle est ce qui permet cette recherche. En psychanalyse, cure analytique et recherche en psychanalyse se confondent : garantir les conditions analytiques de la cure et d'une relation transférentielle est fondamental pour que puisse avoir lieu une recherche. Il n'y a donc pas ici instrumentalisation du cas à visée de recherche, mais visée clinique d'abord, pour assurer les conditions de possibilité de cette recherche. Il convient alors de reposer à nouveaux frais la question : quelle est donc la validité du cas dans la recherche en psychanalyse? Comment le comprendre hors de la psychopathologie, de l'exemplarité, ou de la confirmation de la théorie? Quel en est le fonctionnement proprement analytique?

\section{$\checkmark$ Construction du cas de l'analyste}

Pour Fédida, les cas sont, pour l'analyste, de puissants moteurs de l'analyse sans fin depuis ce lieu de l'autre, producteur de l'auto-analyse. Plus encore, le choix du cas à rapporter en supervision est en étroite liaison avec l'organisation psychique de l'analyste et ses propres points aveugles. Ce choix renseigne sur le contre-transfert, désignant en négatif le lieu de l'impossible réponse de l'analyste à l'analysant/e : il pourrait même être compris comme réaction thérapeutique négative (Fédida, 1995, p. 263). La supervision cherche donc à formuler « l'énigme du cas depuis ce point de sue extérieur à la cure et prenant en compte dans la parole de l'analyste les aveuglements (contre-)transférentiels, y compris ceux de l'analyste superviseur " (ibid., p. 252). Comme le souligne Fédida, au même titre que le rêve ou le mot d'esprit, le cas est une formation de l'inconscient. Il actualise ainsi les fantasmes de l'analyste, qui devient, à travers son récit, analysant/e, sujet désirant. S’il/elle s'acharne à l'écrire, c'est probablement parce qu'il l'a troublé/e : son écoute, ses habitudes théoriques, ses croyances et son expérience clinique sont défaites. C'est là ce que soutient par exemple Theresa de Lauretis, en écrivant, au sujet de “ la jeune homosexuelle » : "C'est l'histoire d'un cas, la reconstruction d'une trajectoire psychique, une 
interprétation, une représentation, un texte de fiction, et non point une " histoire vraie ». C'est un texte qui porte l'inscription d'une subjectivité, un désir, qui sont bien plus ceux de son écrivain, Freud, que ceux de son personnage central, nommé ou non " ${ }^{[15]}$.

Freud pointe probablement cela en désignant, d'un côté, la contradiction consistant à publier des histoires de cas ${ }^{[16]} \mathrm{et,} \mathrm{de} \mathrm{l'autre,} \mathrm{la} \mathrm{compromission} \mathrm{de} \mathrm{l'analyste} \mathrm{dans} \mathrm{cette}$ publication $^{[17]}$. L'écriture de cas est alors tentative de recouvrer une consistance, une identité d'analyste mise en péril par les mouvements transférentiels. Charge à l'analyste de faire qu'elle ne devienne pas rigidification réactionnelle de cette identité, qui, menacée par la déconstruction (et c'est là le mouvement même de l'analyse), s'empresse de se fixer aux stéréotypes de faire l'analyste. L'histoire de cas est ainsi histoire de l'analyste, bien plus que de l'analysant/e, et, pourrait-on ajouter, histoire de l'analyste qui se trompe. Dans son texte de 1937, Freud souligne, en effet, que l'absence totale de réaction de l'analysant/e devant la construction de l'analyste (ni confirmation ni infirmation, juste indifférence) signe l'erreur de cette interprétation. Gageons ici que le cas n'ayant pas l'analysant/e comme allocataire, étant donc dépourvu de toute réaction, signifie que l'analyste se trompe toujours en le rédigeant. Somme toute, comme l'écrivait Freud à un autre égard : “ nul ne peut être mis à mort in absentia ou in effigie " ${ }^{[1]}$. Plus encore, et comme le souligne Fédida, le récit de cas ne devient analytique que dans l'échange entre analystes autour de ce cas : le cas n'existe pas d'emblée, il s'y construit. Un cas n'est alors pas tant la reconstitution d'une histoire, que la construction d'un lieu psychique, depuis le symptôme de l'analyste $^{[19]}$. Le cas apparaît ainsi comme une mise en figure, au sein de “ l'avènement d'une communauté analytique ", rendant parallèles la situation psychanalytique et la construction métapsychologique. En ce sens, la topique d'un cas est lisible en abîme dans la spécularité du texte métapsychologique. En radicalisant cette position, l'écriture du cas ne prendrait ici une forme métapsychologique qu'à éviter de raconter, thématiser, événementialiser directement l'histoire de l'analysant. L'écriture du cas serait ce qui de la situation clinique transparaît dans la théorisation analytique.

[15] Lauretis, 1999, p. 38.

[16] " II est certain que les malades n'auraient jamais parlé s'ils avaient pensé à la possibilité d'une exploitation scientifique de leurs aveux et c'est tout aussi sûrement en vain qu'on leur aurait demandé l'autorisation de les publier. Des personnes scrupuleuses aussi bien que des personnes timides, dans ces conditions, mettraient au premier plan le devoir de la discrétion médicale, et regretteraient de ne pouvoir rendre service à la science en cette circonstance en l'éclairant. Toutefois, je suis d'avis que le médecin a des devoirs non seulement envers le malade, mais aussi envers la science [...]. La publication de ce qu'on croit savoir sur la cause et la structure de l'hystérie devint un devoir, l'omission, une lâcheté honteuse, à condition cependant d'éviter un préjudice direct à son malade » (Freud, 1954, p. 2).

[17] "La discrétion est incompatible avec un bon exposé d'analyse ; il faut être sans scrupule, s'exposer, se livrer en pâture, se trahir, se conduire comme un artiste qui achète les couleurs avec l'argent du ménage et brûle les meubles pour chauffer le modèle. Sans quelques-unes de ces actions criminelles on ne peut rien accomplir correctement » (Freud, 1966, p. 74). [18] Freud, 1912/1953, p. 60.

[19] «En psychanalyse, le cas est une théorie en germe, une capacité de transformation métapsychologique. Le cas est donc partie intégrante d'une activité de construction telle que l'analyse de supervision est en mesure de la constituer. Autrement dit, le cas est construit. En tant que tel, il ne peut relever d'un récit. II n'y a pas d'histoire de cas ! » (Fédida, 1995, pp. 260-261). 
Cette construction du cas n'est pas sans convoquer un parallélisme avec les “ constructions dans l'analyse » de Freud, qui décide alors d'une vérité toute particulière du cas. L'importance de l'affectivité dans la séance est soulignée par Freud dans ce texte de 1937 : l'analysant/e “ doit se souvenir de certaines expériences et des motions affectives suscitées par elles, les unes et les autres se trouvant oubliées à présent ” (Freud, 1985, p. 270). Le travail analytique porte sur ces motions affectives qui, mises à jour, déterminent l'élaboration psychique, bien plus que sur des représentations particulières remémorées. Les souvenirs surgissant chez l'analysant sont comparés par Freud à la fois aux hallucinations ou aux formations délirantes d'une part, pour leur provenance d'une " poussée vers le haut du refoulé », et aux constructions de l'analyste, d'autre part, qui, telles le délire, présentent un morceau de vérité historique. Cette vérité advient non pas par conformation d'une représentation à un contenu, mais par les mouvements affectifs qu'elle provoque. Plus que la littéralité de la construction ou ses contenus, ce sont ses effets affectifs qui importent: la construction n'est qu'une activité préparatoire pour la perlaboration, elle ne vise pas à remplir de remémoration, mais à lever la répétition du transfert. Gageons alors que dans la construction du cas, le contenu narratif n'importe pas non plus : la vérité historique du cas tient davantage à l'effet affectif et, partant, perlaboratif que cette construction opère à la fois sur l'analyste et sur la théorisation.

Plus encore, il semble pertinent de ramener cette vérité historique qui apparaît dans l'élaboration et les effets affectifs de l'écriture de cas, aux conditions discursives de la vérité propre à une époque, à des représentations, à des catégories historiquement inscrites, et à des formations discursives. Celles-ci ne sont que les formes, ponctuelles, qu'emprunte la théorisation hic et nunc, à un moment donné et dans une aire culturelle particulière.

\section{$\checkmark$ Le style du cas : la métaphore}

Si la construction du cas est, comme le souligne Fédida, une " écriture du figurable », les techniques narratives du cas et ses procédés de figuration ouvrent sur la fiction, le double sens et le non-sens liés à la fonction métaphorique du langage, et sur le gain de plaisir découlant de cette activité de figuration. Dans ce fictionnement propre au cas, et constitutif du fonctionnement de la théorie analytique, la métaphore est centrale. Freud prévient en effet à plusieurs reprises de son utilisation métaphorique et par esquisses de la langue de la théorisation, dans laquelle une foison d'hypothèses peut être présentée et accompagnée de jugements critiques, sans toutefois “ prendre l'échafaudage pour le bâtiment lui-même » (Freud, 1967, p. 456).

L'usage de la métaphore est alors particulièrement récurrent chez Freud ${ }^{[20]}$. La métaphore, littéral portage, procède au transport de l'élaboration propre à l'expérience

[20] Citons, à titre d'exemple, les métaphores auxquelles il a recours pour bâtir une théorisation sur le cas de Dora: la surdétermination du symptôme de la toux est exprimée par " ce grain de sable autour duquel les ostracées forment la perle " 
de la cure dans la théorisation. Elle vient garder celle-ci de la folie d'une rigidification dogmatique jamais interrogée, tout autant que d'une élucubration rhapsodique dont le mouvement, sous couvert d'un souci de forme-sens, reprendrait celui de l'inconscient. Elle permet d'inscrire la théorie analytique comme dissolution, déconstruction, per via di levare, plus que comme institution. Elle vient la prévenir du noyau pulsionnel, passionnel, propre à l'infantile du/de la théoricien/ne, du puntum caecum qui agit comme son axe narcissique. Comme le note Fédida dans Topiques de la théorie, si, dans la métaphysique classique, le concept est la non-métaphore par excellence, c'est l'inverse qui a lieu dans la métapsychologie, où l'activité métaphorique est réintroduite à l'intérieur du concept (Fédida, 1978, p. 268). La métaphore permet enfin de reproduire, dans la théorie, la désignification propre à l'espace analytique : jeu de déconstruction des sens liés au cadre socio-culturel, à son inscription historique, ses croyances, ses modèles de comportement, son code symbolique, et ses modalités de perception. En effet, comme le note Jean Cohen, la métaphore introduit une rupture dans l'ordre syntagmatique qu'elle compense par un réagencement de l'ordre paradigmatique (Cohen, 1966). C'est alors par désignification lexicale, délittéralisation, qu'un concept - pulsion, plaisir, narcissisme, sexualité, etc. - est produit en théorie analytique, et c'est par la métaphore qu'il traduit un rapport d'écoute de l'analysant/e.

\section{$\checkmark$ Conclusion : critique de l'écriture de cas}

Qu'est-ce que la critique? Foucault en place le foyer dans « le faisceau de rapports qui nouent l'un à l'autre, ou l'un aux deux autres, le pouvoir, la vérité et le sujet » (Foucault, 2015, p. 39). C'est ainsi que la critique est à concevoir comme “ art de n'être pas tellement gouverné ${ }^{[21]}$. La critique est la mise en question de gouvernementalités. C'est à une critique de l'utilisation du cas en psychanalyse que j’ai tenté ici de me livrer, critique portant sur une dogmatisation de cet exercice de style et des conclusions auxquelles il peut aboutir, sur l'inféodation des cas à une théorie pré-établie, sur son fonctionnement comme cas-paradigme pour la psychopathologie, ou sur sa valeur uniquement pédagogique et illustrative. S’il est en effet pure construction, le cas est également susceptible d'être déconstruit : quelle est alors sa capacité à supporter d'être déformé par une autre théorie que la sienne? Quelle pertinence ont des cas qui ne résistent pas à la déconstruction de leur théorie ? La critique, en outre, est aussi ce qui porte sur une gouvernementalité particulière :

(Freud, 1954, p. 61) ; infections, maladies vénériennes, inflammations et dégoût sont des pensées inconscientes « tendues sur des rapports organiques préfigurés, comparables à des guirlandes de fleurs tendues sur un fil de fer, de sorte qu'on peut trouver, dans un autre cas, d'autres pensées entre les mêmes points de départ et d'arrivée » (Freud, 1954, p. 62) ; enfin, pour rendre compte des associations du rêve de Dora, Freud fait état de " cercles concentriques » autour de la représentation " mouillé », partant de la masturbation pour passer par l'incontinence (mouillé-souillé), la leucorrhée, le catarrhe, et, par fixation de Dora sur la zone orale, le dégoût, la toux et l'enrouement (Freud, 1954, pp. 64-68).

[21] Foucault, 2015, p. 37. 
celle de l'allégeance à une technique et un style analytiques où le récit de cas, l'histoire de cas, le compte rendu d'analyse vont de soi et ne sont pas interrogés dans leur posture énonciative. Qui y parle, serait-on enclin/e à demander, et à qui sont-ils destinés? Qui en est l'allocutaire? Comment alors ne pas être trop gouverné/es, en restant psychanalystes? Probablement en maintenant l'ironie de la “ jeune homosexuelle ", et, partant, ce qui est identifié par Claude Barazer comme son désaveu. Le désaveu se conjugue ici en deux sens :

- loin d'être celui d'une structure perverse de la “ jeune homosexuelle », c'est celui, propre à bien des analystes, qui s'accordent à reconnaître que le cas est fiction, mais ne l'en conçoivent pas moins comme vérité généralisable. Ce désaveu pourrait être illustré par ces formulations : “Je sais bien qu'il s'agit d'une fiction de l'analyste mais quand même, le cas désigne une structure irréductible de la pathologie. »

« Je sais bien que l'histoire de cas est prise dans une économie transférentielle, mais quand même, je la présente comme vérité du/de la patient/e indépendante du (contre)transfert de ma lecture. ”

- En outre, le désaveu est au fondement de la pensée métaphorique. Il s'agit ici de pousser l'ironie jusqu'au bout: la laisser investir la lecture et l'écriture des cas. L'ironie serait ainsi au fondement de la réception du récit de cas et de la théorie qui en résulte. Si un cas est écrit, ce cas comme la métapsychologie restent des fictions : il convient de ne pas trop y croire. Le cas apparaît alors comme un pis-aller théorique, lié à la gageure d'une théorie psychanalytique, s'astreignant à rendre compte de processus primaires par des processus secondaires qui les rateront toujours.

Si l'intérêt du cas est d'agir dans l'écriture analytique, d'y être transporté, métaphorisé, il est alors pertinent de se demander si l'écriture du cas n'a pas à se passer complètement du récit du cas, si l'on ne gagne pas à dépasser, plus que la personne " réelle " du cas, sa narrativité même dans la construction de la théorie analytique. Le cas, la perlaboration qu'il a permise à l'analyste, s'inscrit à même l'écriture analytique, dans la métapsychologie. Nul besoin alors de raconter l'événementialité d'une vie, de communiquer le cas en lui-même, hors supervision, si on ne lui confère pas une valeur démonstrative, ou pédagogique, mais élaborative, celle qui fait qu'il devient métapsychologie.

La clinicographie reste donc un champ purement problématique : celui consistant à ériger des questions, mais en préalable à l'élaboration d'une métapsychologie, où la narrativité du cas n'apparait qu'en creux. Si l'écriture du cas révèle, comme le note Guenaël Visentini, une triple dimension théorique, clinique et politique, force est d'admettre que pour favoriser la visée clinique et politique, le plan théorique a charge d'invisibiliser le cas : d'en annuler la visée de démonstration, d'exemplarité ou d'universalisation. L'écriture de cas se ferait donc sans narration du cas, et viserait alors l'art du cas nul, pour éviter celui du canular. 


\section{* Références}

Ayouch, T. (2015). L'injure diagnostique. Pour une anthropologie de la psychanalyse. In CulturesKairós, $\mathrm{n}^{\circ} 5$ : L'inconscient freudien: débats et pratiques. En ligne sur: http://revues.mshparisnord.org/cultureskairos/index.php?id=1055.

Barazer, C. (1999). “ Ah, mais c'est très intéressant! ” ou l'ironie de la jeune homosexuelle. In Fédida, P. et Villa, F. (dir.) (1999). Le Cas en controverse. Paris : PUF, pp. 103-120.

Bourdellon, G. (2003). La relation homosexuelle, une révélation divine dans une vie de chien. In Revue française de psychanalyse, vol. 67, pp. 95-112.

Cohen, J. (1966). Structure du langage poétique. Paris : Flammarion.

Fédida, P. (1995). Construction du cas. In Le Site de l'étranger. Paris : PUF, pp. 245-266.

Fédida, P. (1978). L'Absence. Paris : Gallimard.

Foucault, M. (1999). Les Anormaux. Cours au Collège de France. 1974-1975. Paris : Gallimard/Seuil. Foucault, M. (2015). Qu'est-ce que la critique? Paris : Vrin.

Freud, S. (1954). Cinq psychanalyses. Paris: PUF.

Freud, S. (1937/1985). Constructions dans l'analyse. In Résultats, idées, problèmes. Tome II. Paris : PUF, pp. 269-286.

Freud, S. (1966). Correspondance avec le Pasteur Pfister 1873-1939. Paris : Gallimard.

Freud, S. (1912/1953). La dynamique du transfert. In La Technique analytique. Paris: PUF, pp. 50-60.

Freud, S. (1900/1967). L'Interprétation des rêses. Paris : PUF.

Freud, S. (1920/1973). Sur la psychogénèse d'un cas d'homosexualité féminine. In Nésrose, psychose, perversion. Paris : PUF, pp. 245-270.

Freud, S. \& Jung, C. G. (1975). Correspondance. Paris : Gallimard.

Gérard, C. (2003). La relation homosexuelle est-elle une relation incestueuse ?. In Revue française de psychanalyse, 2003/1, vol. 67, pp. 113-128.

Lacan J. (1975). Le Séminaire, Livre I. Les écrits techniques de Freud. Paris : Seuil.

De Lauretis, T. (1999). Letter to an unknown woman. In Lesser, R. C. \& Schoenberg, E. That Obscure Subject of Desire : Freud's Female Homosexual Revisited. New York : Routledge, pp. 37-53.

Melman, C. (2002). Que peut nous apprendre aujourd'hui le cas de la jeune homosexuelle? In Cliniques méditerranéennes, 2002/1, nº 65, pp. 69-77.

Pickmann C.-N. (2010). Le cas de Freud dit « La jeune homosexuelle ». In Figures de la psychanalyse, 2010/1, n 19, pp. 195-216.

Pommier, G. (2002). Quelques conséquences du concept de " désistement » pour baliser le champ des homosexualités. In Cliniques méditerranéennes, 2002/1, nº 65, pp. 79-93.

Thouvenin, D. (1999). Propriété/propriétés du cas en psychanalyse. In Fédida, P et Villa, F. (1999). Le Cas en controverse. Paris : PUF, pp. 103-120. 